\section{Critical Historiography and the Design Studio Pedagogy}

Amir H. Ameri

University of Colorado, Denver
The cultures that in their divergent multiplicity were once effectively segregated in space and time, find themselves in close proximity, dialogue and potential competition and conflict in both literal and virtual space as a direct consequence of globalization. Coupled as globalization is with the technologies of the information age, it has dramatically and fundamentally transformed our cultural and cross-cultural modes of communication and exchange, and along with it our cultural experience of space and time. These transformations are not formal and aesthetic perse, but more profoundly cultural and ideological. As such, they are measurably changing all cultures involved in unforeseeable directions. These changes, along with a multi-cultural context to architectural practice in a global economy require a shift of emphasis in architectural pedagogy to better prepare the next generation of architects to meet the unique demands of a plurality of cultures in a state offlux and change.

Assuming that culture and architecture are indispensably linked, and architecture serves, among other cultural mechanisms, to transform our ideas, assumptions, and beliefs about the world into a factual experience of them, what is pedagogically imperative in the face of globalization and rapid cultural change is first and foremost a heightened and at that critical understanding of the complex dialogue between culture and architecture.

The unique challenges of practice in a global market place further mandate a pedagogical shift from the traditional emphasis on the acquisition of bodies of knowledge to fostering analytical, critical, and creative abilities that are not necessarily and always culture specific, i.e., the a bility to a nalyze, organize and manipulate $v$ arious bodies of knowledge in place of their mere amassment. Given the speed and changing modalities of global communication and cross-cultural exchange, bodies of knowledge in their cultural specificity face obsolescence with increased pace, leaving analytical, critical, and creative abilities as the only viable option for keeping pace and/or anticipating cultural change.

The history of Architecture has and will continue to have an indispensable role to play in any curriculum that seeks to instill a heightened understanding of the interconnectedness of architecture and culture. Yet, to play a pivotal role in fostering a spirit of critical exploration and innovation, architectural history has to engage and exert a critical impact on studio pedagogy and that not merely as a repository of formal and aesthetic precedents to justify reiterate choices.

Since secular institutional building-types are the predominant focus of the design studio instruction, engaging the history of their development and revealing their cultural and ideological underpinnings systematically and critically can establish a strong complimentary link between architecture history and design pedagogy. To demonstrate, I'll use the movie-theatre as a c ase study and outline the pedagogy of a design studio that has this building type as its focus.

Assuming that architecture is necessarily and always a theoretical construct, that every edifice inevitably speaks of a thesis regarding itself specifically (including the cultural conditions of its conception and production) and architecture broadly (including the cultural conditions of architecture's conception and definition), students are required at the outset of the studio to ask, research, 
and analyze not what patent 'theory' or 'idea' should their movie-theater speak of, but what arcane theory does its type historically hide under the rubrics of 'function' or 'practical' requirements? What ideology, in other words, does the type refuse to acknowledge as theory in the name of practicality? To find an answer, students are required to do extensive guided research in order to reconstruct the genealogy of the movie theatre as a building type - the genealogy of forms inseparable from the genealogy of the institution served. Students are guided to analyze and critically evaluate the historic role the type plays in establishing and effecting a given institutional/social order as the natural, and practical order of things.

\section{1}

In as much as the movie theater insinuates itself, as it has from inception and perforce, between the real world outside and the imaginary world unfolding on the screen inside, it inevitably locates and localizes the real and the imaginary at a pronounced physical distance. The modalities of this pronouncement define and articulate the perceived relationship between the real and the imaginary. The historic changes in the modalities of this pronouncement have stemmed, in turn, from the often technologically driven changes in the perceived relationship between the real and the imaginary.

Before the advent of movie theaters, the initial and perhaps the most profound change in the relationship of the real and the imaginary happened with the invention of cinema itself. The addition of motion to photographic reproduction further and dramatically altered the preconceived distance between the real and the imaginary to the point of a spatial, if not ideational crisis. Inasmuch as film overlaps and condenses time and space, it inherently displaces every place it happens to be. It produces a strange cohabitation between heterogeneous spaces, past and present, real and illusory, virtual and actual. The ensuing sense of displacement is well documented in early reactions to film exhibition, coming as they did before the advent of the movie theater. ${ }^{1}$

The challenge of (dis)locating and keeping film at a safe distance, was first met at the Nickelodeon. Despite its short history, Nickelodeon was to have a profound influence on the history of movie theaters in the century to come. Whereas literally, if not in effect, cinema brings other spaces and times to our space and time and as such creates a potentially uncanny cohabitation, the designers of the Nickelodeon effectively sidestepped this challenge by turning the experience on its head, conceptualizing it as a journey out to an other place. To this end, the designers of Nickelodeon focused primarily on fabricating a thick borderline between the world outside and the screen placed at the far end of the auditorium furthest, both conceptually and literally, from that world. The process of instituting an other space for film often began, as David Hulfich explained in 1913, with the conversion of a vacant store. ${ }^{2}$ The transparent glass façade was removed and replaced with an opaque wall placed at some distance from the street façade. Over the latter was superimposed a gateway imagery whose ubiquity made it in short order synonymous with the
Nickelodeon. If the movie theater is, as Mary Heaton Verse noted in 1911, "the door of escape, for a few cents, from the realities of life," this escape - n o less from reality - was not merely imaginary. ${ }^{3}$ It was also a literal experience that was enacted architecturally and ritually to the estrangement of narrative cinema from every place it happened to be.

\section{2}

The development and ensuing popularity of feature-length movies in the e arly teens brought with it an important shift in the relationship of the audience to the filmic event. Soon the Nickelodeon was declared "obsolete and altogether unsuited" to the exhibition of feature-length movies. ${ }^{4}$ The cause was the obsolescence of Nickelodeon's localization in face of g reater intensity and duration of involvement with the imaginary. Rapp aptly attributed the shape of things that became to a new vision for what the movie theater ought to be in face of rapidly improving film productions:

A second period in the history of the motion picture theater began - with the advent in the field of a different type of showman - o ne who believed that people go to the theater to live an hour or two in a different world; that the atmosphere of a palace should prevail in a theater, and that this could be arrived at by gorgeous stage settings, luxurious drapes and enchanting music. ${ }^{5}$

Of course, this new vision was not entirely new. What it had in common with the old is rendering the movie-going experience a journey out to an other place. However, whereas the Nickelodeon's primary focus was the institution and elaboration of a threshold in between the real and the imaginary, the Movie Palaces of the silent era focused on fabricating a "different world" beyond the Nickelodeon's threshold, literally. Film was now to happen in a world apart, where exoticism, and in short order, orientalism was to underscore a difference that was not only visceral, but also dramatic and literal.

Thomas Lamb, whose work for Marcus Loew also played a seminal role in shaping the history of the Movie Palace, succinctly articulated the strategy for this "new" motion picture theater in 1928:

To make our audience receptive and interested, we must cut them off from the rest of the city life and take them into a rich and self-contained auditorium, where their minds are freed from their usual occupations and freed from their customary thoughts. In order to do this, it is necessary to present to their eyes a g eneral scheme quite different from their daily environment, quite different in color scheme, and a great deal more elaborate. ${ }^{6}$

The sources of the movie palace decoration were as diverse as European aristocratic palaces from one end to a vast and diverse repertoire subsumed under the label "Orient" to the other. All that mattered was exoticism and other-worldliness "conspiring to create an effect thoroughly foreign to our Western minds," 
thereby casting "a spell of the mysterious and to the Occidental mind exceptional." ${ }^{7}$ In this exotic and Oriental imaginary, the moviegoers were transformed into visiting tourists in a foreign, displaced, and displacing land, where film stood in the same relationship to the real as Orient did to Occident. Here, the imaginary was not per se what the movie brought to its place; it was a reception the place imposed on the movie in advance.

Led on by succeeding vistas through successive spaces, the sight-seeing "adventure" of the audience/tourist culminated in the auditorium. Although the style and the details varied, what Movie Palace auditoria shared in common was richly articulated wall surfaces that decisively enveloped the auditorium as a centralized space that located and localized the audience in the requisite "rich and self-contained" place.

An important measure of the auditorium's requisite self-containment as a p lace was the elaborate and o rnate proscenium arch erected as a monumental threshold at the far end of the auditorium, opposite the entry doors. It produced two distinct and segregated spaces, that localized the audience and the imaginary in their respective and mutually exclusive places at an unabridged distance on the two sides of the proscenium arch.

However novel, strange, and/or engrossing the displacement of time and space behind the proscenium arch may have been, at every draw of the curtain, one inevitably found oneself at a distance from both the event and the illusory enveloping veneer of an exotic destination, that wasn't. Here, in an other world designed to be looked at, o ne was never let in, though all the while inside.

\section{3}

It would not be until the early 30's that the initial technological challenges of adding sound to movies, including synchronization and sound quality, would be overcome, the novelty would wear off, and "talkies" would become merely movies. In the process, the relationship of the audience to the filmic event would undergo a profound transformation and along with it the movie-theater whose function remained the ideational sublimation of that relationship. What would also remain constant is the strategic journey out to a n elsewhere.

Although the changes the movie-theater underwent in the 1930's had everything to do with sound, it had not to do with acoustics per se. The Movie Palace auditoria were acoustically superior to the movie auditoria that replaced them. The change had to do with the abridgment of the distance between the audience and the imaginary, and a deliberate attempt to reestablish the distance.

Much as sight takes cognizance of distance, sound overcomes and collapses distance. It is heard and felt here, where the listener happens to be, rather than there, at the source. Reaching the audience from across the multiple thresholds erected in the Movie Palace auditoria to keep the filmic event at a safe and spectatorial distance, the talkies, transformed spectators into voyeurs. The defenses built to date against the uncanny effect of film proved no defense against sound.

To reestablish the abridged distance between the real and the imaginary, all the trappings of exoticism and orientalism were dropped in short order to transform the movie-theater from an exotic destination into a path to an imaginary destination. Ben Schlanger who played an instrumental role in shaping the new movie-theater summed up his lifetime effort in 1961:

The desire in the designing was to permit the viewer to the fullest possible extent to be able to transport himself in imagination to a different time and space by furnishing a floating void or optical vacuum to provide the transition to the new time and space and to hold him there by e liminating all distractions. The name Transcenium suggests itself ....8

The audience would hereby never be given to arrive in a literal and literally exotic place. They would remain on a path and in "transport," as it were, to and from an imagined and imaginary destination before and after the filmic event. Through the "floating void" of the new auditorium, sound would no longer be given to reach the audience in any place identifiable as such, exotic or otherwise. Instead, the placeless "optical vacuum" of the new "Transcenium" would "transport" the audience to its imaginary place for the duration of the filmic event. B efore and after, the audience would remain on a path through a "floating void" to and from no place real.

\section{4}

As color film overcame yet another divide between the real and the imaginary and went from being an exception to becoming norm in the 50's and early 60's, the movie-theater was transformed yet again to reestablish the abridged distance between the real and the imaginary. This time the logic of the Movie Palace was conjoined to the logic of the "Transcenium" theater as the movie-theater was (re)moved to a new profoundly segregated world dedicated to spectatorship: the mall. To reach the new "Transcenium" theater, one now had to travel to a new and "different world" through roads, across a sea of parking segregating it, not unlike a moat, from its surrounding environment, only to arrive at an indoor outdoor space, where the passage of time and the vagaries of weather and seasons were suspended in a theatrical space dedicated to exhibition and spectatorship. Here, everyone was transformed into a spectator/tourist away from home in an exaggerated version of the Movie Palace's exotic alterity, long before embarking on a temporal journey through the "floating void" of the auditorium to an imaginary destination.

From here on, were the movie-theater not to depend on a mall, it would fabricate its own mall in front of the "Transcenium" theater, as Multiplexes have and 
continue to do.

5

If cinema is indeed a response to what Benjamin referred to in 1936 as "the desire of contemporary masses to bring things 'closer' spatially and humanly," the history of cinema's place and placement has followed the opposite trajectory. ${ }^{9}$ Much as ambivalence persistently overshadows any question of a d ecidable place for film, nevertheless, a persistent spacing has kept film at bay from inception.

In effect, and at face value, the objective has been to keep the real and the imaginary at a pronounced distance. This has not been for fear of unbridled cohabitation, or any possibility of confusion between the real and the imaginary per se. Rather at issue in the absenting of each from the construed place of the other has been the clarity of the line separating the real from the imaginary, i.e., their radical alterity.

The reasoning is perhaps best alluded to in Freud's essay on the uncanny. "An uncanny effect," Freud noted in 1919, "is often and easily produced by effacing the distinction between imagination and reality, ... or when a symbol takes over the full functions and significance of the thing it symbolizes, and so on." ${ }^{10}$ A case in point, Freud noted, is confusing one's own reflection for someone real and o ther than o neself. The uncanny sensation has not to do with the confusion as such. Rather, the sensation is associated with the recognition of the confusion after the fact, i.e., the recognition of having momentarily and involuntarily taken the imaginary for the real. Regarding the cause of the sensation, Freud notes:

This uncanny is in reality nothing new or foreign, but something familiar and old-established in the mind that has been estranged only by the process of repression. This reference to the factor of repression enables us, furthermore, to understand Schelling's definition of the uncanny as something which ought to have been kept concealed but which has nevertheless come to light. ${ }^{11}$

What in the uncanny is familiar and repressed, and ought to have been kept concealed, is not the substitution, rather it is the condition of its possibility. It is the possibility of the distinction between the real and the imaginary being the function and the effect of spacing, i.e., extrinsic rather than intrinsic to the real and the imaginary alike. It is the repressed recognition that what is imagined and imaginary is the line separating the real and the imaginary, as the condition of the possibility of substitution and/or confusion.

For the image to be separable and transportable, and at that subject to involuntary substitution, it must be always separable and transportable already, in origin, as it is in every repetition. Cinema's dispensation with the presence of the referent as the point of origin-without the loss of pretense to objective representationbrings to surface a gap between the visual and the substantive contents of reality. This gap between form and substance, or image and identity, may be covered but never bridged. The exposure of this gap offers a serious challenge to the privileged antecedence and alterity of reality as measured against the imaginary. Cinema subjects the aura of humanist reality to radical query insofar as the possibility of its fabrications and the proximity of its representations strip reality of its endowed authority as the site of a causal link between form and substance, or image and identity. The visual content of the real can only be made to precede and be independent of its actual substantive content in the imaginary world if the two had not a causal, but a conventional relationship in the real. Cinema can only give visual content spatial and temporal mobility if reality that is always rigorously distinguished from the imaginary is itself already a form of representation. Subject as it is to cinema's manipulative interventions and imaginary doubling that forgo the possibility of a site for causality, humanist reality stands to disappear as a selfsame entity, only to surface as a suppressed imaginary and a purposed construction, always already. Otherwise, there could be no signification without a present referent. In "authentic reality," as in the "illusion of reality" the referent is perpetually deferred. The self has never been but in exile from the "reality," that is never given, and always desired.

That "authentic reality" is, in a sense, always already an "illusion of reality," i.e., divided and deferred and as such a substitute for a desired reality that is undivided and fully p resent to itself is "nothing new or foreign, but familiar and old-established in the mind that has been estranged only by the process of repression." That the difference between "authentic reality" and "illusion of reality" is also an indifference is what ought to "have been kept concealed but which has nevertheless come to light" in the figure of the uncanny.

If the question of the film's place and placement has loomed large since its inception, it is, in no small measure, a reflection of the problematically undifferentiated and undifferentiable space of the imaginary. The imaginary at once exceeds and defies any sense of place or any act of placement, predicated upon, in the simplest terms, a clear boundary separating two opposite terms, e.g., here and there, inside and outside. The imaginary has no outside, since outside every p resumed or presumable place for it, o ne only finds the imaginary.

However, to institute and protect the aura of the real as the self-referential, non-representational other of the imaginary, the movie-theatre, as an institution and a b uilding type, has systematically fabricated an outside to the imaginary, if only to locate and safeguard the real at a safe distance. This is not because the two are diametrically opposed. Rather, it is because any perceived line separating them is always a construct and never a given. As an institution and a building type, the movie-theatre substitutes a formal, s patial, a nd experiential clarity of distance for the very s patial and temporal dimensions that cinema as the imaginary fundamentally places in question. The institution of the movie-theatre has been an instituted resistance to the subversive, exposing effect of the imaginary on the real as the presumed and imagined self-referential, non-representational other of the imaginary.

The intent in engaging the history of the movie-theatre as a secular building type, coupled with a systematic and critical 
re-evaluation of its ideational presuppositions at the outset of the design studio is twofold. The first intent is to help students develop the type of analytical and critical skills that are requisite to deciphering the intricate relationship between architectural form, function and ideology. The second intent is to explore how a critical historiography of secular building types can foster a spirit of exploration, experimentation, critical engagement, creative thought and innovation, that are necessary skills for architects in the global information age. To this latter end, a c ritical historiography of secular building types readily serves as an analytical foundation for a studio pedagogy that does not ask students to reproduce either the form or the logic of the type. The critical re-evaluation of the building type readily forms the parameters of a n ew context for design, within which the link between the formal/architectural properties of the building type and its institutional/cultural presuppositions could neither be acknowledged nor ignored, neither reinforced nor discarded. A c ontext within which there could be no intuitive and/ or positive re-formulation of the building type in affirmation of the link, but only a critical formation in recognition of the link.

What, for instance, students are asked, would a movie-theatre be like, that did not try to sublimate the imaginary, but recognize its undecidable nature, i.e., both the difference and the indifference, the similarity and the alterity of the imaginary and the real. If the imaginary defies any borderlines and the clarity of any distinction and separation from the real, can something of the same logic be taken to forming its place. If the movie-theatre as we know it substitutes a clear distinction between a host of spatial and formal dichotomies - center and periphery, path and place, container and contained, interiority and exteriority - can one conceive and design a movie-theatre whose formal and experiential properties do not lend themselves to or support the conception of the imaginary as the other of the real. In short, can one design a building that poses questions instead of offering preconceived answers? Can one design a building that offers no singular, determinate reading and no experiential dichotomies as such? The pedagogical intent of such a d esign challenge is to p romote a c onscious re-evaluation of all the subconscious assumptions regarding spatial organization, the relationship of parts to whole, the inside to the outside, the particulars of volume and mass, solid and void, path and place, s tructure and material, ornamentation, proportion, scale, and others.

To this end, students begin the design p rocess by analyzing and understanding the dual nature of various architectural element as both a f unction and an expression, i.e., in terms of what each does and what each says or is capable of expressing. They are given design exercises that require them to distinguish and explore how architecture communicates both statically and dynamically, in space and in time, i.e., passive and active reception. They are asked to distinguish between experiencing architecture, which is accumulative, and viewing it, which is totalizing as a mode of reception. They are asked to recognize and shape space as the fundamental experiential element of architecture as well as how to use form as a means to shaping space as o pposed to an end in itself.

Students are asked, for instance, to design a wall that is neither simply transparent nor simply opaque but both at once. Such a design problem cannot be approached literally. Each term is literally exclusive of the other. Transparency and opacity must be broached as perceptual and experiential qualities that pertain to the expressed relationship between the spaces in front and behind the dividing wall. The latter's is merely the medium of the articulation of that relationship, in address to a viewing subject. The wall can, in time, be multiplied and transformed into a room intended to read as neither finite nor infinite, but both at once. This is a room or a space where the viewing subject's perception oscillates between the readings of expansion and contraction, stasis and movement, without resolution. The ambivalent experiential qualities of this room, in turn, can be brought to the design of the movie auditorium where the encounter with the imaginary is to ultimately take place. This is an auditorium whose o scillating experiential qualities is intended to parallel the medium it houses. In tandem, the same design strategies can be taken to locating and forming the movie-theater as a whole in relationship to its context, i.e., neither connected nor disjoined, neither a part nor apart from that context. Movie-theater as the space of the imaginary in relation to the context as the place of the real.

In sum, the pedagogical strategy outlined above is intended to foster a tangible understanding of the crucial interplay between analysis and design as two complementary processes. It is meant to guide students to understand analysis as a process of moving from realization to abstraction (e.g., from form to principle, to intent) and design as a process of going from abstraction to realization (e.g., from intent, to strategy, to form). This is with the intention of designing a building that in the end is all too familiar and yet all too alien, one that is neither a copy nor strictly an original. A building that speaks silently of the designer's ability to willfully manipulate the language of architecture as opposed to faithfully re-produce its various speech acts. This last is, perhaps, the most essential skill in the global information age.

\section{Notes}

1. See: Gorky, Maxim, "A review of the Lumiere programme at the Nizhni-Novgorod Fair," in Kino: A History Of The Russian And Soviet Film, ed. Jay Leyda (Princeton: Princeton University Press, 1983), 407-09

2. Mary Heaton Verse, "Some Picture Show Audiences." Outlook 98 ( June 24, 1911): 442

3. David Hulfish, Motion-Picture Work: A General Treatise on Picture Taking, Picture Making, PhotoPlays, and Theater Management and Operation (Chicago: American Technical Society), 12-45

4. George L. Rapp, "History of Cinema Theater Architecture," in Living Architecture, ed. Arthur Woltersdorf (Chicago: A. Kroch, 1930), 58. In addition see P.R. Pereira, "The Development of the Moving Picture Theater," American Architect 106, (1914): 178

5. Rapp, History of Cinema, 59

6. Thomas W. Lamb, "'Good Old Days" to these Better New Days," Motion Picture News (June 30, 1928): 14

7. Lamb, "Good Old Days," 14

8. Ben Schlanger, "Motion-Picture System From Camera to 
Viewer," The Society of Motion Picture and Television Engineers Journal 70, no. 9 (September 1961): 685

9. Walter Benjamin, "The Work of Art in the Age of Mechanical Reproduction," in Illuminations (New York: Schocken Books, 1978), 222

10. Sigmund Freud, "The Uncanny," in Studies in Parapsychology (New York: Collier Books, 1977), 50

11. Freud, The Uncanny, 47 